\title{
Serum Soluble Glycoprotein VI (sGPVI) to Predict the 28th Day In-Hospital Mortality in Adult Patients with Sepsis
}

Sabry Abd Allah Shoeib ${ }^{1}$, Ayman Fathy Abd ElHalim², Mohamad Ahmad Abd ElHafez ${ }^{1}$, Ashraf Abd ElRaof Dawod ${ }^{3}$, Essam Ali Abd EIMohsen ${ }^{4}$, Sameh Abo EINasr Abd ElSalam ElBaz ${ }^{1 *}$

${ }^{1}$ Department of Internal Medicine and Hematology, ${ }^{3}$ Department of Medical Biochemistry, Faculty of Medicine, Menoufia University, ${ }^{2}$ Department of Internal Medicine and Hematology, Faculty of Medicine, Zagazig University, ${ }^{4}$ Department of Internal Medicine and Hematology, El Maadi Military Hospital, Egypt

*Corresponding author: Sameh Abo ElNasr Abd El Salam ElBaz, Mobile: (+20)1006896109, E-Mail: samehelbaz@ymail.com

\begin{abstract}
Background: Sepsis is a complicated syndrome resulting from the inappropriate expression of host factors in response to infection and is a major cause of death in patients that are hospitalized in emergency departments and in critical care units.

Objective: The purpose of the present study was to identify the role of serum soluble glycoprotein VI (sGPVI) in predicting the 28th day in-hospital mortality in adult patients with sepsis.

Patients and methods: This observational prospective cohort study included a total of 90 adult patients aged $\geq 18$ years old with clinically suspected sepsis, recruited from Medical Intensive Care Units of Menoufia University Hospital and Kafr El-Sheikh general hospital. The included subjects were diagnosed using quick SOFA score and according to survival at the 28th day, they were divided into two groups; Group A (survivors) consisted of 69 patients (76.7\%) and Group B (non survivors) consisted of 21 patients (23.3\%).

Results: As regard clinico-laboratory data (qSOFA, sGPVI, SGOT, respiratory rate, ESR, WBCs) and complications (hospital stay), they are significantly increased (P value $<0.001$ ) in non-survivor studied patients in comparison with survivor ones. Concerning platelet count, it is significantly reduced ( $\mathrm{P}$ value $<0.001$ ) in non-survivor studied patients in comparison with survivor ones. As regard clinico-laboratory data (qSOFA, SGOT, respiratory rate, ESR and WBCs) and complications (hospital stay), they are positively correlated with sGPVI with a statistically significant difference in non-survivor studied patients. Concerning platelet count, it is negatively correlated with sGPVI with a statistically significant difference in non-survivor studied patients.
\end{abstract}

Conclusion: It could be concluded that glycoprotein VI is a valuable prognostic predictor during sepsis being more reliable than other widely used indicators and severity scores, such as qSOFA, WBCs, platelet count and CRP.

Keywords: Sepsis, sGPVI, qSOFA, CRP.

\section{INTRODUCTION}

Sepsis has been redefined as life-threatening organ dysfunction caused by dysregulated host responses to infection and septic shock as a subset of sepsis in which particularly profound circulatory, cellular and metabolic abnormalities are associated with a greater risk of mortality than with sepsis alone ${ }^{(\mathbf{1})}$.

Sepsis cases represent either a new organ dysfunction or worsening of chronic organ dysfunction such as those seen in comorbid conditions in the context of suspected or proven infection ${ }^{(2)}$.

The literature on prevalence of organ dysfunction outside the critical care environment is limited and when estimated appears frequently ${ }^{(3)}$. Alongside this underestimated incidence globally, the short-term mortality from sepsis is improving (4).

Essentially, the sepsis response is triggered by an invading pathogen, usually of bacterial origin but also viral or fungal, or a pathogen-produced substance, such as endotoxin, called a pathogen-associated molecular pattern. These bind to pattern recognition receptors (PRRs) present on macrophages, polymorphonuclear and endothelial cells to initiate the release of proinflammatory mediators that cause vasodilatation and changes in endothelial cell function resulting in altered blood flow distribution, increased vascular permeability and tissue oedema in an attempt to limit the spread of the infection. Coagulation, complement and contact systems are also activated, further promoting the inflammatory response. Endogenous danger-associated molecular patterns (such as heatshock proteins) are also released and interact with PRRs to promulgate the inflammatory response ${ }^{(5)}$.

The biomarkers of sepsis can be classified as markers of acute-phase protein (C-reactive protein [CRP], procalcitonin [PCT], lipopolysaccharidebinding protein), cytokine/ chemokine biomarkers interleukin (IL-6),(IL-8), and markers of other pathophysiologic processes (coagulation factors and soluble cell surface receptors). Currently, complement factors $(\mathrm{C} 3 \mathrm{a}, \mathrm{C} 5 \mathrm{a}$, and the soluble form of the C5a receptor, $\mathrm{sC} 5 \mathrm{aR}$ ) have been defined as early markers of sepsis and sepsis severity. CRP and PCT are the most

This article is an open access article distributed under the terms and conditions of the Creative Commons Attribution (CC BY-SA) license (http://creativecommons.org/licenses/by/4.0/) 
widely used for the detection of blood stream infections (6).

Platelet glycoprotein VI (GPVI) represents an attractive new target as an antiplatelet reagent, because it is only expressed on platelets and platelet precursor cells in bone marrow (megakaryocytes), and GPVI blockade has demonstrated efficient antithrombotic potential in the experimental models of thrombosis without enhancing pathological bleeding ${ }^{(7)}$.

The aim of the current work was to identify the role of serum soluble glycoprotein VI (sGPVI) in predicting the 28th day in-hospital mortality in adult patients with sepsis.

\section{PATIENTS AND METHODS}

This observational prospective cohort study included a total of 90 adult patients aged $\geq 18$ years old with clinically suspected sepsis, recruited from Medical Intensive Care Units of Menoufia University Hospital and Kafr El-Sheikh general hospital. This study was conducted between January 2019 till January 2020. Written informed consent of all the subjects was obtained.

\section{Ethical approval:} obtained.

\section{Approval of the ethical committee was}

The included subjects were diagnosed using quick SOFA score and according to survival at the 28th day, they were divided into two groups; Group A (survivors) consisted of 69 patients $(76.7 \%)$ and Group B (non survivors) consisted of 21 patients (23.3\%).

\section{All participants were submitted to:}

- Full history taking: included age, sex, family history and any other medical or surgical history.

- Complete clinical examination: included general complete examination and estimation of quick Sequential Organ Failure Assessment (qSOFA) score of 3 points which comprises altered mentation (Glasgow Coma Scale - GCS <15), systolic blood pressure $(\leq 100 \mathrm{mmHg})$ and respiratory rate $(\geq 22$ breaths/min).

- Investigations: Routine laboratory tests as complete blood count (CBC), C reactive protein (CRP), erythrocyte sedimentation rate (ESR), serum creatinine, random plasma glucose, liver enzymes, and urine analysis.

\section{Sampling and procedures:}

Under complete aseptic conditions, $5 \mathrm{~m} 1$ venous blood samples were withdrawn from the antecubital vein using disposable plastic syringe after sterilization of skin with iso propyle alcohol $(70 \%)$ swabs obtained from each patient. $2 \mathrm{ml}$ were added to EDETA for complete blood count (CBC) using computerized method. $1 \mathrm{~m} 1$ was collected in plain plastic tube to separate serum which was kept at $-20^{\circ} \mathrm{C}$ until time of $\mathrm{C}$ reactive protein (CRP) assaying. $2 \mathrm{ml}$ blood were collected into vacutainers containing 0.106 $\mathrm{nM}$ sodium citrate as anticoagulant $(10 \% \mathrm{vol} / \mathrm{vol})$ for glycoprotein analysis. Platelet poor plasma was prepared by centrifugation of whole blood at $2000 \mathrm{~g}$ for 10 minutes. Plasma was aliquoted and stored at $-80^{\circ} \mathrm{C}$ until analysis.

\section{Interpretation of Results:}

Agglutination in the highest serum dilution corresponds to the approximate amount of CRP in $\mathrm{mg} / \mathrm{dl}$ present in the test specimen.

Concentration of CRP calculated as follows:

$$
\mathrm{CRP}(\mathrm{mg} / \mathrm{dl})=\mathrm{S} \times \mathrm{D}
$$

Where, $S=$ Sensitivity of the reagent i.e. $0.6 \mathrm{mg} / \mathrm{dl}$. $\mathrm{D}=$ Highest dilution of serum showing agglutination .

\section{Measurement of soluble glycoprotein (sGPVI): according to Connolly-Anderson et al. ${ }^{(9)}$.}

GPVI levels were measured by immunoassay. 96 well standard binding plates from MesoScale Discovery (MSD, Rockville, MD) were coated overnight at $4^{\circ} \mathrm{C}$ with $4 \mu \mathrm{g} / \mathrm{mL}$ human GPVI polyclonal antibody (R\&D Systems, Abingdon, UK). The plate was blocked with 5\% MSD Blocker A for 1 hour at RT, washed x 3 with $150 \mu \mathrm{L}$ PBS / $0.05 \%$ Tween (PBST) and $25 \mu \mathrm{L}$ of undiluted platelet poor plasma added to duplicate wells. Samples were incubated at RT with vigorous shaking for 1 hour. The plate was washed $\mathrm{x} 3$ with PBST. Biotinylated human GPVI antibody was diluted to $1 \mu \mathrm{g} / \mathrm{mL}$ in $1 \%$ MSD Blocker A and $25 \mu \mathrm{L}$ added to each well. The plate was incubated at RT for 1 hour with shaking at $650 \mathrm{rpm}$ then washed $\mathrm{x} 3$ with PBST. $150 \mu \mathrm{L} 2 \mathrm{x}$ read buffer was added to each well and the plate read on a MesoScale Quickplex SQ120 Plate Scanner according to the manufacturer's instructions. The intra- and inter-assay coefficients of variation $(\mathrm{CVs})$ for sGPVI were 4.9 and $5 \%$ respectively with normal range up to $200 \mu \mathrm{g} / \mathrm{ml}$.

\section{Statistical methods}

Data were coded and entered using the statistical package for the Social Sciences (SPSS) version 26 (IBM Corp., Armonk, NY, USA). Data was summarized using mean and standard deviation for quantitative variables and frequencies (number of cases) and relative frequencies (percentages) for categorical variables. Comparisons between groups were done using unpaired $\mathrm{t}$ test. For comparing categorical data, Chi square $(\chi 2)$ test was performed. Exact test was used instead when the expected frequency is less than 5. Correlations between quantitative variables were done using Pearson correlation coefficient. ROC curve was constructed with area under curve analysis performed to detect best cutoff value of sGPVI for prediction of death. P-values 
less than 0.05 were considered as statistically significant.

\section{RESULTS}

As regard clinico-laboratory data (qSOFA, sGPVI, SGOT, respiratory rate, ESR, WBCs) and complications (hospital stay), they are significantly increased ( $P$ value $<\mathbf{0 . 0 0 1})$ in non-survivor studied patients in comparison with survivor ones. Concerning platelet count, it is significantly reduced (P value < 0.001) in non-survivor studied patients in comparison with survivor ones (table 1).

Regarding comorbidities (HTN) and complications (ventilation), they are statistically significant ( $P$ value $<0.001$ and 0.002 respectively) in non-survivor studied patients in comparison with survivor ones (table 2).

As regard clinico-laboratory data (qSOFA, SGOT, respiratory rate, ESR and $\mathrm{WBCs}$ ) and complications (hospital stay), they are positively correlated with sGPVI with a statistically significant difference in nonsurvivor studied patients. Concerning platelet count, it is negatively correlated with sGPVI with a statistically significant difference in non-survivor studied patients (table 3).

Concerning the area under the receiver operating characteristic (ROC) curve of sGPVI results, it was of 1.00 (95\% CI 1.00-1.00) and a cutoff value of sGPVI is $945 \mathrm{ug} / \mathrm{ml}$ with a sensitivity of $100 \%$ (table 4 \& figure 1).

Table (1): Comparison of demographic (age), clinico-laboratory data and complications (hospital stay) between survivor and non-survivor studied patients.

\begin{tabular}{|c|c|c|c|c|c|}
\hline & \multicolumn{4}{|c|}{ Survival } & \multirow{3}{*}{$P$ value } \\
\hline & \multicolumn{2}{|c|}{\begin{tabular}{|l|} 
survivor \\
\end{tabular}} & \multicolumn{2}{|c|}{ non-survivor } & \\
\hline & Mean & $\begin{array}{l}\text { Standard } \\
\text { Deviation }\end{array}$ & Mean & $\begin{array}{l}\text { Standard } \\
\text { Deviation }\end{array}$ & \\
\hline GOT & 50.13 & 3.46 & 53.64 & 2.63 & $<0.001 *$ \\
\hline Creatinine (mg/dl) & 1.95 & 0.32 & 2.01 & 0.40 & 0.526 \\
\hline sGPVI (ng/mL) & 620.35 & 115.17 & 1224.29 & 169.96 & $<0.001 *$ \\
\hline RR & 23.13 & 1.46 & 24.62 & 1.77 & $0.002 *$ \\
\hline Temperature & 38.66 & 0.46 & 38.46 & 0.31 & $0.023 *$ \\
\hline HR & 109.65 & 8.59 & 107.86 & 6.74 & 0.324 \\
\hline ESR (mm/hr.) & 70.22 & 8.68 & 86.86 & 4.85 & $<0.001 *$ \\
\hline CRP (mg/L) & 48.65 & 8.21 & 62.29 & 3.16 & 0.084 \\
\hline Hb (g/dl) & 10.96 & 0.84 & 10.84 & 0.69 & 0.504 \\
\hline WBCs $\left(x^{10} 0^{9} / 1\right)$ & 14.71 & 1.29 & 22.30 & 3.21 & $<0.001 *$ \\
\hline $\begin{array}{l}\text { Platelet count } \\
\left(\mathbf{x 1 0}^{9} / \mathrm{l}\right)\end{array}$ & 180.09 & 14.64 & 79.00 & 10.68 & $<0.001^{*}$ \\
\hline Age (years) & 62.96 & 2.65 & 63.86 & 2.15 & 0.120 \\
\hline $\begin{array}{l}\text { Hospital stay } \\
\text { (days) }\end{array}$ & 4.16 & 0.80 & 7.19 & 1.08 & $<0.001 *$ \\
\hline $\begin{array}{l}\text { qSOFA } \\
(\geq 2-\leq 3)\end{array}$ & 2.06 & 0.24 & 2.90 & 0.30 & $<0.001 *$ \\
\hline
\end{tabular}

(*) statistically significant. 
Table (2): Comparison of demographic data (sex), comorbidities and complications (bacteremia and ventilation) between survivor and non-survivor studied patients.

\begin{tabular}{|c|c|c|c|c|c|c|}
\hline \multirow{3}{*}{\multicolumn{2}{|c|}{ Data }} & \multicolumn{4}{|c|}{ Survival } & \multirow{3}{*}{ P valu } \\
\hline & & \multicolumn{2}{|c|}{ survivor } & \multicolumn{2}{|c|}{ non-survivor } & \\
\hline & & Count & $\%$ & Count & $\%$ & \\
\hline \multirow{2}{*}{ Sex } & Male & 40 & $58.0 \%$ & 13 & $61.9 \%$ & \multirow{2}{*}{0.748} \\
\hline & Female & 29 & $42.0 \%$ & 8 & $38.1 \%$ & \\
\hline \multirow{2}{*}{ Bacteremia } & Positive & 42 & $60.9 \%$ & 15 & $71.4 \%$ & \multirow{2}{*}{0.379} \\
\hline & Negative & 27 & $39.1 \%$ & 6 & $28.6 \%$ & \\
\hline \multirow{2}{*}{ CVD } & Positive & 59 & $85.5 \%$ & 18 & $85.7 \%$ & \multirow{2}{*}{1} \\
\hline & Negative & 10 & $14.5 \%$ & 3 & $14.3 \%$ & \\
\hline \multirow{2}{*}{ Ventilation } & Positive & 13 & $26.1 \%$ & 18 & $61.9 \%$ & \multirow{2}{*}{$0.002^{*}$} \\
\hline & Negative & 51 & $73.9 \%$ & 8 & $38.1 \%$ & \\
\hline \multirow{2}{*}{ CKD } & Positive & 53 & $76.8 \%$ & 14 & $66.7 \%$ & \multirow{2}{*}{0.351} \\
\hline & Negative & 16 & $23.2 \%$ & 7 & $33.3 \%$ & \\
\hline \multirow{2}{*}{ CLD } & Positive & 62 & $89.9 \%$ & 18 & $85.7 \%$ & \multirow{2}{*}{0.693} \\
\hline & Negative & 7 & $10.1 \%$ & 3 & $14.3 \%$ & \\
\hline \multirow{2}{*}{ CHD } & Positive & 52 & $75.4 \%$ & 18 & $85.7 \%$ & \multirow{2}{*}{0.385} \\
\hline & Negative & 17 & $24.6 \%$ & 3 & $14.3 \%$ & \\
\hline \multirow{2}{*}{ HTN } & Positive & 18 & $36.2 \%$ & 25 & $85.7 \%$ & \multirow{2}{*}{$<0.001^{*}$} \\
\hline & Negative & 44 & $63.8 \%$ & 3 & $14.3 \%$ & \\
\hline \multirow{2}{*}{ DM } & Positive & 41 & $59.4 \%$ & 12 & $57.1 \%$ & \multirow{2}{*}{0.853} \\
\hline & Jegative & 8 & $0.6 \%$ & & $2.9 \%$ & \\
\hline
\end{tabular}

(*) statistically significant.

Table (3): Correlation between sGPVI and demographic (age), clinico-laboratory data and complications (hospital stay) of all studied patients.

\begin{tabular}{|l|l|l|l|}
\hline \multirow{2}{*}{} & SGPVI & P value & N \\
\cline { 2 - 4 } & $\mathbf{R}$ & $<0.001^{*}$ & 90 \\
\hline SGOT & 0.384 & 0.373 & 90 \\
\hline Creatinine (mg/dl) & 0.095 & $0.003^{*}$ & 90 \\
\hline RR & 0.305 & 0.416 & 90 \\
\hline Temp & -0.087 & 0.064 & 90 \\
\hline HR & 0.196 & $<0.001^{*}$ & 90 \\
\hline ESR (mm/hr.) & 0.508 & 0.371 & 90 \\
\hline CRP (mg/L) & 0.095 & 0.694 & 90 \\
\hline Hb\% (g/dl) & -0.042 & $<0.001^{*}$ & 90 \\
\hline WBCs (x10 $/ \mathbf{l})$ & 0.727 & $<0.001^{*}$ & 90 \\
\hline Platelet count (x10 $\boldsymbol{\text { /l) }}$ & -0.853 & $<0.001^{*}$ & 90 \\
\hline Hospital stay (days) & 0.924 & $<0.001^{*}$ & 90 \\
\hline qSOFA & 0.808 & 0.062 & 90 \\
\hline Age years) & 0.197 & & \\
\hline
\end{tabular}

(*) statistically significant.

Table (4): Cutoff value, sensitivity and specificity of sGPVI.

\begin{tabular}{|c|c|c|c|c|c|c|c|}
\hline & \multirow{2}{*}{\begin{tabular}{|c} 
Area Under \\
the ROC \\
Curve
\end{tabular}} & \multirow[b]{2}{*}{$P$ value } & \multicolumn{2}{|c|}{ 95\% Confidence Interval } & \multirow[b]{2}{*}{ Cut off } & \multirow[b]{2}{*}{$\begin{array}{c}\text { Sensitivity } \\
\%\end{array}$} & \multirow[b]{2}{*}{$\begin{array}{c}\text { Specificity } \\
\%\end{array}$} \\
\hline & & & Lower Bound & $\begin{array}{l}\text { Upper } \\
\text { Bound }\end{array}$ & & & \\
\hline sGPVI & 1.000 & $<0.001 *$ & 1.000 & 1.000 & 945 & 100 & 100 \\
\hline
\end{tabular}




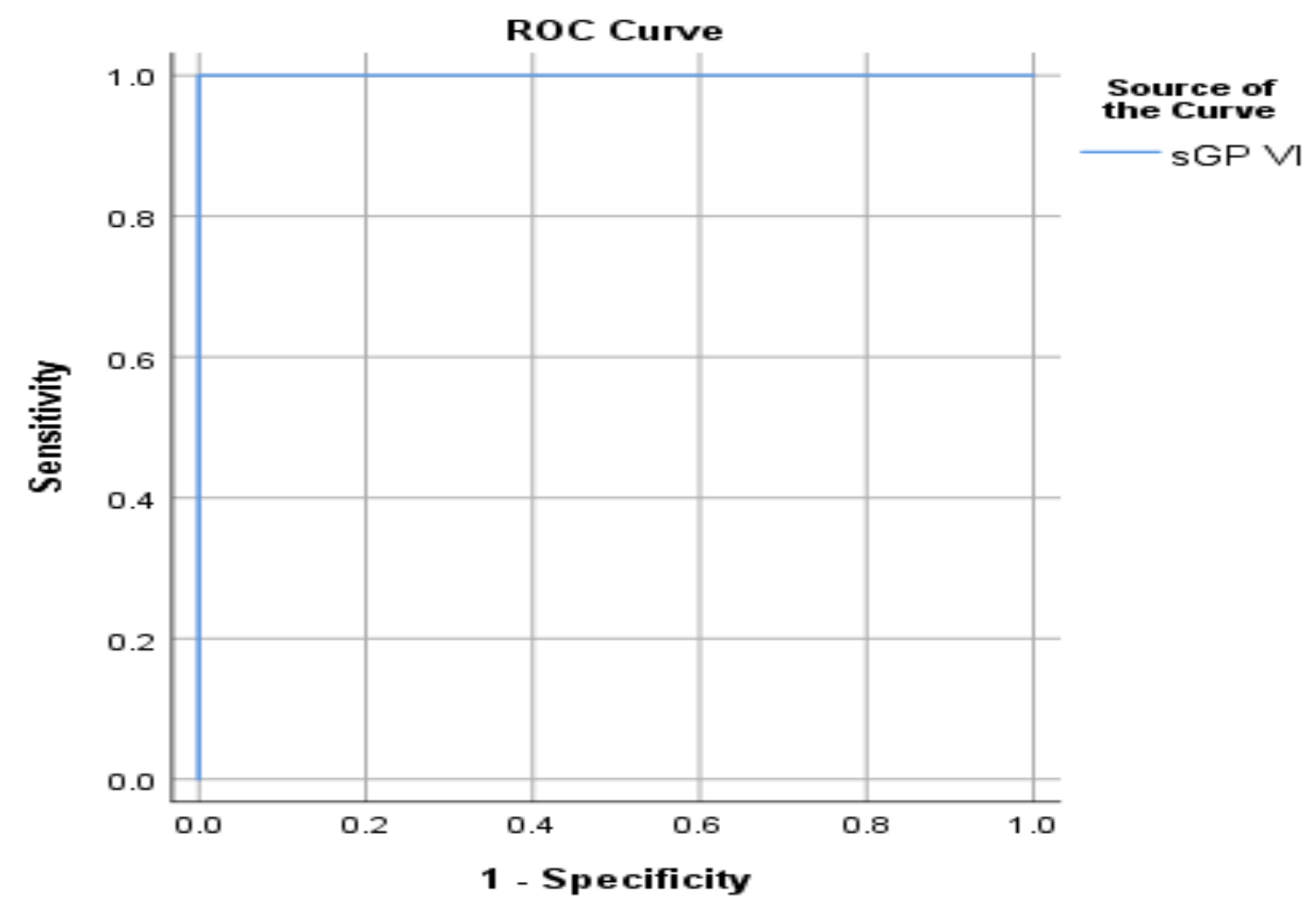

Figure (1):- ROC curve of sGPVI.

\section{DISCUSSION}

In the present study in patients with sepsis criteria, temperature and heart rate were nearly comparable between survivors and non-survivors associated with increased use of mechanical ventilator in non-survivor patients. Regarding quick Sequential Organ Failure Assessment score (qSOFA), respiratory rate and hospital stay, they increased significantly in non-survivor patients. Seigel et al. ${ }^{(10)}$ reported that non-invasive technique had identified critically ill patients by recognition of body temperature patterns. Fever as a classic symptom of septic patients has minor sensitivity and specificity in relation to diagnostic expressiveness. Dupuy et al. ${ }^{(11)}$ showed that abnormal body temperature curves were predictive of the diagnosis of sepsis in afebrile critically ill patients. Analysis of temperature patterns may relieve the decision to antimicrobial therapy rather than absolute temperature values.

Measurement of the heart rate variability (HRV) is a promising technique to evaluate the autonomic cardiac regulation in patients with suspected sepsis. Although underlying mechanisms are still unclear, changes in HRV are associated with the appearance of systemic infections and correlate with disease severity ${ }^{\mathbf{( 1 2})}$. The measurement of HRV may be a promising tool to improve an early diagnosis of sepsis. In adult bone marrow transplant patients, alterations in HRV could be detected prior to the clinical diagnosis of sepsis (13). Regarding comorbidities, coronary heart diseases (CHD), diabetes mellitus (DM) and hypertension (HTN) increased significantly in patient with sepsis. These results agreed with Jimenez-Ibanez et al. ${ }^{(14)}$ who reported that shortterm hyperglycemia is an independent risk factor for mortality in patients without a past medical history of DM. The blood glucose level of patients with DM is usually higher than that of healthy people, which may make them better able to tolerate the impact of shortterm hyperglycemia when sepsis occurs. Conversely, when non-diabetic patients with sepsis experience short-term hyperglycemia, circulating inflammation cytokine levels increase significantly ${ }^{(\mathbf{1 5})}$.

As regard laboratory data of patient with sepsis criteria hemoglobin and bacteremia were nearly comparable between survivors and non-survivors in these patients. Creatinine, CRP and GOT increased significantly in non-survivor groups when compared with survivor patients. WBCs and ESR increased significantly in non-survivor patient when compared with survivor patient associated with significant decrease of platelet count. These results agreed with Hsiao et al. ${ }^{(16)}$ who observed that WBCs and CRP increased in patients with sepsis

Rashwan et al. (17) reported that CRP increased in survivor patients with non-statistical significant differences.

C-reactive protein is widely used in the critical care setting, and its value as a prognostic marker is proven in many diseases including sepsis ${ }^{\left({ }^{(18)}\right.}$. Serum levels of CRP nearly comparable between survivors and non survivors in patient with sepsis ${ }^{(\mathbf{1 9})}$. Seligman et al. (20) suggested that decreasing CRP values between the onset and fourth day of sepsis could predict survival. This difference may owing to a large number of patients (75 patients), measurement of the 
CRP every day, and calculation of the change in the CRP level. The duration of the study was 4 days only.

CRP is commonly used to screen early onset of sepsis. High CRP levels correlate with disease severity. Although CRP assessment does not sufficiently allow to discriminate between infectious and non-infectious stimuli, a secondary rise in CRP level after 3 to $4 \mathrm{~d}$ after infection, surgery or trauma could be helpful for diagnosing septic complications ${ }^{(21)}$.

Orati et al. (22) reported that patients with abdominal sepsis had significantly higher serum CRP levels. One possible explanation for this phenomenon is surgical trauma in the patients with abdominal sepsis. Surgical trauma causes significant increases in CRP levels compared with the preoperative values, especially on the second day after the trauma, even in non-infected patients; thus, such a mechanism may be responsible for the higher CRP values in abdominal sepsis ${ }^{(23)}$.

The measurement of circulating megakaryocyte precursors provides a good indicator of megakaryocytopoiesis, and hence platelet production in sepsis. Thrombopoietin (Tpo) is the principal physiologic regulator of megakariocytopoiesis and platelet production. The circulating Tpo levels were found to be high in the face of low platelet counts in sepsis. Immune cells recognize pathogens through Toll-like Receptors (TLRs). The TLRs allow platelets to recognize bacterial proteins during sepsis and regulate platelet immunity and function ${ }^{(24)}$.

In non-survivor patient mechanical ventilation intervention increased significantly when compared with survivor patients. Moreover, Glycoprotein VI increased significantly in non-survivor patient when compared with survivors. These results agreed with Hsiao et al. ${ }^{(16)}$ who reported that a significantly higher percentage of mechanical ventilation in non-survivors than survivors $([95 \% \mathrm{CI}]: 4.76[1.62-13.9], \mathrm{p}$ value $=$ 0.01 ) and this may indicate an increase in sepsis severity. Mechanical ventilation may therefore be considered a risk factor or indicator of sepsis severity. It is used to prevent lung collapse and decrease sepsisinduced acute lung injury, acute respiratory distress syndrome, and acute respiratory failure in severe sepsis (25).

Hematologic scoring system (HSS) can improve the diagnostic accuracy of complete blood count. It can be employed as a screening test for diagnosing sepsis. But it is important to simplify and standardize the interpretation of this global test ${ }^{(26)}$.

Also, agreed with Montague et al. ${ }^{(27)}$ who noticed that glycoprotein VI increased in patient with sepsis. During sepsis, Glycoprotein promotes the antiinflammatory response by binding to the adhesion molecule L-selectin, decreasing neutrophil migration and rolling, granulocyte extravasation, and recruitment of T-cells to the endothelium or of platelets to the infection site, and modulating nitro oxide-dependent pathways and glycan moiety composition (28).

In studies done by Barroso et al. ${ }^{(29)}$ and Hsiao et al. (16), GP level was significantly correlated in patients with sepsis upon admission. AGP is hepatocyte-secreted positive acute phase proteins, with the former playing an important role in human infection. It exert protective effects as plasma concentrations increase during early sepsis ${ }^{(30)}$.

In a dynamic analysis of GP levels in different outcomes, we observed that GP concentrations differed significantly at different time points in survivors and non-survivors and that patients who died showed higher GP concentrations. This is because GP exerts protective effects during early sepsis, but may become damaging if high levels are maintained over an extended period of time, resulting in immune-paralysis during the later phase of sepsis ${ }^{(31)}$.

In the present study, sGPVI increased significantly in non-survivor patient when compared with survivors and being nearly comparable to other traditional prognostic indicators of sepsis (CRP, WBCs, qSOFA score and platelet count). This results agreed with Brinkman-van der Linden et al. ${ }^{(32)}$ who demonstrated that non-surviving septic patients displayed a marked elevation of fucosylated GP and increased expression of sialyl Lewis X (SLeX) groups on GP, suggesting that these changes in GP glycosylation may provide prognostic value in sepsis

sGPVI has been shown to inhibit the rolling/adhesion and migration of leukocytes and the adhesion and aggregation of platelets. These functions are related to the direct interaction of its glycan moiety, especially its sialyl Lewis X (sLeX) residue, with selectins present in leukocytes and platelets ${ }^{(33)}$.

If high levels of AGP are present for extended periods of time, it is possible that this can lead to immunoparalysis, which is related to increased rates of nosocomial sepsis, MODS and death in critically ill patients ${ }^{(34)}$. Previously, in murine models of sepsis, their group has demonstrated that administration of GP can inhibit the migration of neutrophils to infectious foci, increasing the mortality of animals (28). Furthermore, Spiller et al. ${ }^{(35)}$ demonstrated that increased AGP concentrations observed during diabetes play a role in the high susceptibility to infection in this disease.

\section{CONCLUSIONS}

It could be concluded that glycoprotein VI plays a vital role for early diagnosis of sepsis, to predict outcome, and to guide choice of antibiotic therapy. It is a valuable prognostic predictor during sepsis being more reliable than other widely used indicators and severity scores, such as qSOFA, WBCs, platelet count and CRP. Glycoprotein VI level measurements meet the major requirements for outcome prediction in 
treating patients with sepsis and increased in nonsurvivor patients.

\section{REFERENCES}

1. Shankar-Hari $\mathrm{M}$, Ambler $\mathrm{M}$, Mahalingasivam $\mathrm{V}$ et al. (2016): Evidence for a causal link between sepsis and long-term mortality: a systematic review of epidemiologic studies. Critical Care, 20(1):1-10.

2. Singer M, Deutschman CS, Seymour CW et al. (2016): The third international consensus definitions for sepsis and septic shock (sepsis-3). JAMA., 315(8):801-808.

3. Churpek MM, Zadravecz FJ, Winslow C et al. (2015): Incidence and Prognostic Value of the Systemic Inflammatory Response Syndrome and Organ Dysfunctions in Ward Patients. Am J Respir Crit Care Med., 1: 1-5.

4. Stevenson EK, Rubenstein AR, Radin GT et al. (2014): Two decades of mortality trends among patients with severe sepsis: a comparative meta-analysis. Crit Care Med., 42(3):625-631.

5. Elias AC, Matsuo T, Grion CM et al. (2012): Incidence and risk factors for sepsis in surgical patients: a cohort study. J Crit Care, 27: 159-166.

6. Charchaflieh J, Rushbrook J, Worah S et al. (2015): Activated complement factors as disease markers for sepsis. Dis Markers, 382-463.

7. Gawaz M, Vogel S, Pfannenberg C et al. (2014): Implications of glycoprotein VI for theranostics. Thromb Haemost., 112(1):1-6.

8. Angus DC, van der Poll T (2013): Severe sepsis and septic shock. N Engl J Med., 369: 840-851.

9. Connolly-Anderson AM, Sundberg E, Ahlm C et al. (2015): Increased thrombopoiesis and platelet activation in hantavirusinfected patients. J Infect Dis., 212(7): 1061-1069.

10. Seigel TA, Cocchi MN, Salciccioli J et al. (2012): Inadequacy of temperature and white blood cell count in predicting bacteremia in patients with suspected infection. J Emerg Med., 42: 254-259.

11. Dupuy A-M, Philippart F, Pe'an Y et al. (2013): Role of biomarkers in the management of antibiotic therapy: an expert panel review: I-currently available biomarkers for clinical use in acute infections. Ann Intensive Care, 3(1):22-29.

12. Barnaby D, Ferrick K, Kaplan DT et al. (2002): Heart rate variability in emergency department patients with sepsis. Acad Emerg Med., 9: 661-670.

13. Ahmad S, Ramsay T, Huebsch L et al. (2009): Continuous multiparameter heart rate variability analysis heralds onset of sepsis in adults. PLoS One, 4: 6642-6645.

14. Jimenez-Ibanez EO, Castillejos-Lopez M, Hernandez A et al. (2012): High mortality associated with hyperglycemia, neutrophilia, and lymphopenia in critically ill patients. Tohoku J Exp Med., 226(3):213-220.

15. Yu WK, Li WQ, Li N et al. (2003): Influence of acute hyperglycemia in human sepsis on inflammatory cytokine and counterregulatory hormone concentrations. World J Gastroenterol., 9(8):1824-1827.

16. Hsiao S, Yun-Ru L, Chia T et al. (2019): $\alpha$-1-Acid Glycoprotein Concentration as an Outcome Predictor in Adult Patients with Sepsis BioMed Research International, 19:1-9.

17. Rashwan NI, Mohammed H, Mohey El-Deen Z et al. (2019): Validity of biomarkers in screening for neonatal sepsis. Pediatrics and Neonatology, 60:149-155.

18. Thiem U, Niklaus D, Sehlhoff B et al. (2009): C-reactive protein, severity of pneumonia and mortality in elderly, hospitalised patients with community-acquired pneumonia. Age Ageing, 38: 693-697.

19. Hillas G, Vassilakopoulos T, Plantza $P$ et al. (2010): Creactive protein and procalcitonin as predictors of survival and septic shock in ventilator-associated pneumonia. Eur Respir J., 35: 805-811.

20. Seligman R, Papassotiriou J, Morgenthaler NG et al. (2008): Copeptin, a novel prognostic biomarker in ventilator-associated pneumonia. Crit Care, 12:11-13.

21. Hofer N, Zacharias E, Müller W et al. (2012): An update on the use of C-reactive protein in early-onset neonatal sepsis: current insights and new tasks. Neonatology, 102: 25-36.

22. Orati JA, Patricia A, Vanessa S et al. (2013): Serum Creactive protein concentrations in early abdominal and pulmonary sepsis. Rev Bras Ter Intensive, 25(1): 12-19.

23. Miyano G, Okazaki T, Kato Y et al. (2010): Open versus laparoscopic treatment for pan-peritonitis secondary to perforated appendicitis in children: a prospective analysis. $\mathbf{J}$ Laparoendosc Adv Surg Tech A.,20(7):655-657.

24. Beaulieu LM and Freedman JE (2010): The role of inflammation in regulating platelet production and function: Toll-like receptors in platelets and megakaryocytes. Thromb Res., 125: 205-209.

25. Peake SL, Delaney A, Bailey M et al. (2014): Goal-directed resuscitation for patients with early septic shock. New England Journal of Medicine, 371(16): 1496-1506.

26. Narasimha A and Harendra Kumar M (2011): Significance of Hematological Scoring System (HSS) in Early Diagnosis of Neonatal Sepsis. Indian J Hematol Blood Transfus., 27(1):1417.

27. Montague SJ, Celine D, Christelle L et al. (2017): Soluble GPVI is elevated in injured patients: shedding is mediated by fibrin activation of GPVI. Blood Advances, 2(3): 240-246.

28. Mestriner FL, Spiller F, Laure HJ et al. (2007): Acute-phase protein alpha-1-acid glycoprotein mediates neutrophil migration failure in sepsis by a nitric oxide-dependent mechanism," Proceedings of the National Acadamy of Sciences of the United States of America, 104(49): 19595-19600.

29. Barroso R, Sousa RR, Mendonc $P$ et al. (2013): Decreased levels of alpha-1-acid glycoprotein are related to the mortality of septic patients in the emergency department. Clinics, 68(8): 1134-1139.

30. Vandevyver S, Dejager L, Vandenbroucke RE et al. (2014): An acute phase protein ready to go therapeutic for sepsis. EMBO Molecular Medicine, 6(1): 2-3.

31. Frazier $W$ and Hall $M$ (2008): Immunoparalysis and adverse outcomes from critical illness. Pediatric Clinics of North America, 55(3): 647-668.

32. Brinkman-van der Linden EC, van Ommen EC, van D (1996): Glycosylation of alpha 1-acid glycoprotein in septic shock: changes in degree of branching and in expression of sialyl Lewis(x) groups. Glycoconj J., 13(1):27-31.

33. Ceciliani F, Pocacqua V (2007): The acute phase protein alpha1-acid glycoprotein: a model for altered glycosylation during diseases. Curr Protein Pept Sci., 8(1):91-108.

34. Frazier W (2011): Immunity, inflammation and sepsis: new insights and persistent questions. Crit Care, 15(1):124-126.

35. Spiller F, Carlos D, Souto FO et al. (2012): alpha1-Acid Glycoprotein Decreases Neutrophil Migration and Increases Susceptibility to Sepsis in Diabetic Mice. Diabetes, 61(6):15841591. 